\title{
Signal-generated memory for different N-lengths: Effects on resistance to extinction
}

\author{
STEVEN J. HAGGBLOOM and DANIEL A. BUFKIN \\ Arkansas State University, State University, Arkansas
}

\begin{abstract}
Three groups of rats $(n=4)$ received runway training in Phase 1 to establish, in each group, one stimulus as a signal for a single nonreinforced $(N)$ trial and another stimulus as a signal for four consecutive $\mathrm{N}$ trials. All groups received consistent reinforcement (CR) in Phase 2, with Group N-4 receiving the signal for four $N$ trials and Group N-1 receiving the signal for one $N$ trial, twice each day. Group CR received no signal presentations in Phase 2. Phase 2 was followed by 20 unsignaled extinction trials, 5 trials per day. As predicted by the signal-generated memory hypothesis, Group N-4 was more resistant to extinction than Groups N-1 and CR.
\end{abstract}

Haggbloom (1988) established a stimulus as a signal for nonreinforcement $(\mathrm{N})$ in an initial phase of discrimination training and then presented that stimulus on selected reinforced $(R)$ trials during a subsequent phase of consistent reinforcement (CR) training. This treatment, in groups labeled Signal-N Groups, substantially increased resistance to extinction relative to various control groups not given signal presentations in Phase 2. The increased resistance to extinction obtained in Signal-N Groups, called the signal-generated partial reinforcement extinction effect (PREE), can be understood as follows.

According to the signal-generated memory hypothesis (Haggbloom, 1988; Haggbloom, Lovelace, Brewer, Levins, \& Owens, 1990), presentation of a signal associated with $\mathbf{N}$ or $\mathbf{R}$ generates retrieval of a temporally remote memory of that outcome. Call the memory of a temporally remote $\mathbf{N}$ or $\mathbf{R}$ activated in this way a signal-generated memory (signal-generated $S^{N}$ and $S^{R}$, respectively), and call the memory of an $\mathbf{N}$ or $\mathbf{R}$ event stored on one trial and retrieved on the next trial an event-generated memory (event-generated $S^{N}$ and $S^{R}$, respectively).

Under the conditions employed by Haggbloom (1988), presentation of a signal associated with $\mathbf{N}$ during $\mathbf{C R}$ training caused signal-generated $S^{N}$ to replace event-generated $S^{R}$ as the operative memory. Because retrieval of $S^{N}$ was generated on an $R$ trial during $C R$ training in Phase 2, subjects were conditioned to respond to $S^{N}$ as though the previous trial had actually been an $\mathbf{N}$ trial, as in actual partial reinforcement (PR) training (see, e.g., Capaldi, 1966). Indeed, Haggbloom (1988, Experiments 4 and 5) reported increased resistance to extinction in Signal-N groups that matched that obtained in groups given actual PR training and conditioned to respond to event-generated $S^{\mathbf{N}}$.

In the experiments reported by Haggbloom (1988), the stimulus established as a signal for $\mathrm{N}$ was associated with a single $\mathrm{N}$ trial. According to Capaldi's (1966) sequen-

Correspondence concerning this article should be addressed to S. J. Haggbloom, P.O. Box 2127, State University, AR 72467. tial theory, different lengths of run of $\mathrm{N}$ trials ( $\mathrm{N}$-lengths) are represented as distinctively different memories, and longer $\mathrm{N}$-lengths experienced in acquisition increase resistance to extinction more so than shorter $\mathrm{N}$-lengths. This is so because $S^{\mathbf{N}}$ associated with long $\mathbf{N}$-lengths bears more similarity to $S^{N}$ present after several extinction trials than does $\mathrm{S}^{\mathrm{N}}$ associated with shorter $\mathrm{N}$-lengths.

The purpose of our experiment was to investigate whether a signal associated with a relatively long $\mathrm{N}$-length would generate greater resistance to extinction than would one associated with a short $\mathrm{N}$-length under signalgenerated memory retrieval conditions otherwise similar to those employed by Haggbloom (1988). We trained three groups of rats under essentially identical conditions in Phase 1, establishing one stimulus as a signal for a single $\mathrm{N}$ trial, and another stimulus as a signal for four consecutive $\mathrm{N}$ trials. In Phase 2 , which consisted of $\mathrm{CR}$ training for all groups, the signal for one $\mathrm{N}$ trial was presented on selected $\mathrm{R}$ trials to Group $\mathrm{N}-1$ and the signal for four $\mathrm{N}$ trials was presented to Group $\mathrm{N}-4$. No signal presentations were experienced in Phase 2 by Group CR. Following Phase 2, all subjects received five extinction trials per day for 4 days. There were no signal presentations during extinction.

\section{METHOD}

\begin{abstract}
Subjects
The subjects were 12 male rats, approximately 90 days old at the start of the experiment, bred in the laboratory from Holtzman stock.

\section{Apparatus}

The apparatus consisted of a single straight alleyway that was $235 \mathrm{~cm}$ long, $10 \mathrm{~cm}$ wide, and $15 \mathrm{~cm}$ high. The alleyway was made of wood and had a hinged hardware cloth top. The initial $25 \mathrm{~cm}$ and final $25 \mathrm{~cm}$ of the runway constituted start- and goalboxes, respectively. The startbox and goalbox were separated from the rest of the runway by manually operated guillotine doors. Three clocks $(.01 \mathrm{sec})$ recorded start, run, and goal times over respective distances of $20 \mathrm{~cm}$, the next $150 \mathrm{~cm}$, and the next $30 \mathrm{~cm}$ from the startbox door. Opening the startbox door activated the first clock, and photoelectric circuitry controlled its offset and the operation of the remaining two clocks. The goalbox contained an unpainted goal cup constructed by drilling a $6-\mathrm{cm}$-diam hole in a
\end{abstract}


small block of wood. The goal cup was positioned against the end wall of the goalbox.

\section{Procedure}

The rats were housed in individual cages with access to water throughout the experiment. Two weeks before the start of training, the rats were placed on food deprivation, receiving $14 \mathrm{~g}$ of lab chow per day. Once runway training began, this food allowance was in addition to food consumed in the runway. On Days 12-14 of deprivation, the rats were handled individually for 2-3 min. After being handled on those days, the rats were fed eight .045-g Noyes Formula A food pellets from a small dish in their home cages. Four rats were randomly assigned to each of three groups, Groups N-1, N-4, and CR.

Phase 1. All three groups received three different reward schedules, one in each of three daily sessions of trials, on each of 18 days of training in Phase 1. Schedule A consisted of four consecutive R trials (.045-g food pellets). Schedule $B$ consisted of $R$ followed by one half of a Kellogg's Corn Puff (P) and then a single N trial (RPN) for Groups N-4 and CR, or R followed by one half of a Kellogg's Cocoa Puff $(C)$ and then a single $\mathrm{N}$ trial $(\mathrm{RCN})$ for Group $\mathrm{N}-1$. Finally, Schedule $\mathrm{C}$ was RCNNNN for Groups N-4 and CR and RPNNNN for Group N-1. There are six possible permutations of Schedules A, B, and C, and each permutation occurred once within each block of 6 days. The intertrial interval within sessions was $10-15 \mathrm{sec}$, and the interval between sessions was at least $15 \mathrm{~min}$.

Phase 2. Each group received five trials per day on each of 5 days in Phase 2 with all trials occurring in a single session. Group CR received $\mathrm{R}$ on each trial, and Groups $\mathrm{N}-1$ and $\mathrm{N}-4$ received the schedules RCRCR (Days 1, 3, and 5), CRRCR (Day 2), and CRCRR (Day 4). In Group N1, $\mathrm{C}$ was associated with a single $\mathrm{N}$ trial, whereas in Group $\mathrm{N}-4, \mathrm{C}$ was associated with four $\mathrm{N}$ trials.

Extinction. Each group received five extinction trials per day for 4 days.

Trial administration procedures. A trial was begun by opening the startbox door approximately 2-3 sec after placing the rat in the apparatus and regardless of the rat's orientation. The rats were run in squads containing 1 rat from each group. The order of running rats within a squad was varied randomly each day; the order of successive squads was held constant across days. On $\mathrm{N}$ trials, the rats were confined to the unbaited goalbox for $20 \mathrm{sec}$. A maximum time of $60 \mathrm{sec}$ was allowed in each section of the runway. If $60 \mathrm{sec}$ were exceeded in any section, the additional time was added to the score of the next section forward. If the rat did not enter the goalbox in $180 \mathrm{sec}$, it was placed in the goalbox.

\section{RESULTS}

All times were converted to speeds in centimeters per second. Only analyses based on total speeds are reported here. Table 1 shows running speeds for each group, averaged over the last four days of Phase 1, on each trial of both Series B and Series C. As can be seen, all groups

Table 1

Running Speeds for Each Group Averaged Over the Last 4 Days of Phase 1 for both the Three- and the Six-Trial Series

\begin{tabular}{ccccccc}
\hline & \multicolumn{7}{c}{ Days } \\
\cline { 2 - 7 } Group & 1 & 2 & 3 & 4 & 5 & 6 \\
\hline \multicolumn{7}{c}{ Six-Trial Series } \\
N4 & 109.23 & 112.45 & 67.43 & 42.85 & 27.61 & 26.53 \\
N1 & 111.50 & 113.73 & 53.48 & 16.36 & 20.40 & 10.42 \\
CR & 113.14 & 113.77 & 38.78 & 25.88 & 20.66 & 17.23 \\
\multicolumn{7}{c}{ Three-Trial Series } \\
N4 & 110.54 & 108.41 & 64.77 \\
N1 & 111.31 & 104.01 & 59.71 & & \\
CR & 115.40 & 113.34 & 54.17 \\
\hline
\end{tabular}

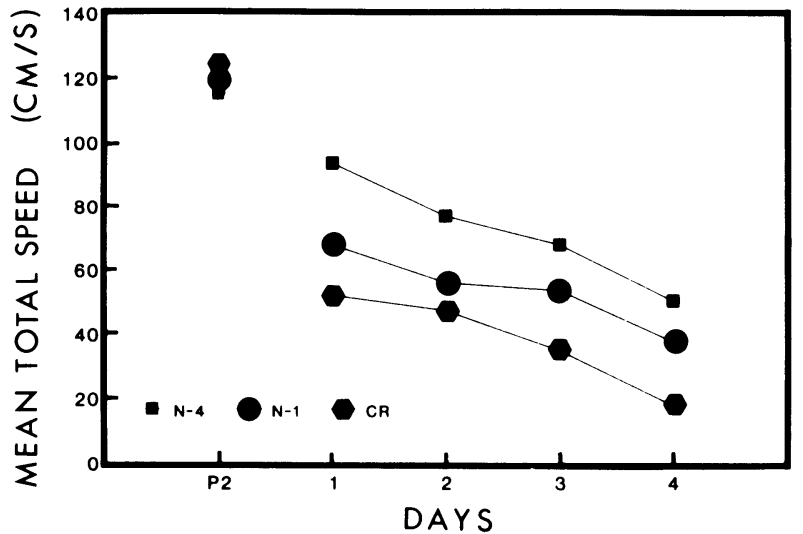

Figure 1. Mean speed for each group on the last day of Phase 2 and on each day of extinction.

learned to anticipate and run slow to the single $\mathrm{N}$ trial of Series B and to the four $\mathrm{N}$ trials of Series C. There were no differences among the three groups in Phase 1. Separate analyses of variance (ANOVAs), with groups (3) as a between-subject variable and trials ( 3 or 6$)$ and days (4) as within-subject variables, were applied to speeds over the last 4 days of Phase 1 . Respectively, both the 3- and the 6-trial ANOVAs yielded highly reliable trials effects $[F(2,18)=88.42$, and $F(5,18)=119.85$, $p s<.001]$, but there were no differences among groups $[F<1$ and $F(2,9)=1.39]$, nor was there a groups $\times$ trials interaction $[F<1$ and $F(10,45)=1.17]$.

Figure 1 shows the mean running speed for each group on the last day of Phase 2 and on each of 4 days of extinction. As can be seen, there were no differences among groups in speed of running at the end of Phase 2. In extinction, Group N-4 ran faster than Group N-1, which in turn ran faster than Group CR. A group (3) $\times$ trial (5) ANOVA applied to the last day of Phase 2 showed there were no significant differences among groups $(F<1)$, nor was there a groups $\times$ trials interaction $(F<1)$. A group (3) $\times$ trial (5) $\times$ day (4) ANOVA applied to extinction speeds yielded a reliable groups effect $[F(2,9)=6.14, p<.05]$. No other terms in the ANOVA approached statistical significance. Planned comparisons of the differences among group means were done with one-tailed tests. As predicted, Group N4 $(M=72.16)$ was more resistant to extinction than Group N1 [M= 53.75; $F(1,9)=3.57, p<.05]$. Group N4 was also more resistant to extinction than Group CR [ $M=38.06$, $F(1,9)=12.25, p<.01]$. The difference between Groups N1 and CR was short of a conventional level of significance $[F(1,9)=2.60, .05<p<.10]$.

\section{DISCUSSION}

Group N-4, given a signal prior to selected $R$ trials during Phase 2 $\mathrm{CR}$ training that was associated in Phase 1 with an $\mathrm{N}$-length of four, was more resistant to extinction than was Group N1, given a signal associated in Phase 1 with a single $\mathrm{N}$ trial. And with the relatively small number of subjects employed here, only Group N-4 showed a reliable signal-generated PREE. 
The greater resistance to extinction in Group $\mathrm{N}-4$ relative to Group $\mathrm{N}-1$ was predicted by the signal-generated memory hypothesis (Haggbloom, 1988). According to that view, signal presentations in Phase 2 served to generate retrieval of the memory of four consecutive $\mathbf{N}$ trials in Group N-4 $\left(S^{N_{4}}\right)$ so that $S^{N_{4}}$ replaced event-generated $S^{R}$ as the operative memory. Group $\mathrm{N}-4$ subjects were thus conditioned to respond to $\mathrm{S}^{\mathrm{N}_{4}}$ as though they had just experienced an $\mathrm{N}$-length of four during PR training. In contrast, signal presentations in Phase 2 generated retrieval of the memory of a single $\mathrm{N}$-trial $\left(\mathrm{S}^{\mathrm{N}_{1}}\right)$ in Group $\mathrm{N}-1$ so that $\mathrm{S}^{\mathrm{N}_{1}}$ replaced event-generated $S^{R}$ as the operative memory. Group $N-1$ subjects were thus conditioned to respond to $S^{N_{1}}$. According to sequential theory (Capaldi, 1966), groups conditioned to respond to memories of longer $\mathrm{N}$-lengths will show greater resistance to extinction than will groups conditioned to respond to memories of relatively shorter $\mathrm{N}$-lengths, a prediction confirmed here with signal-generated memories.

The signal-generated memory effects reported here resulted from the use of a procedure that was subtly different from that employed by Haggbloom (1988) and Haggbloom et al. (1990). In the earlier experiments, the signal was not a component of the reinforcement schedule (e.g. a pretrial feeding, tactile stimulus, or brightness stimulus). In the present experiment, qualitatively different reinforcements obtained on one tria signaled either one or four subsequent $\mathbf{N}$ trials. In both situations, however, a signal that was extrinsic to the target memory was used to generate memory retrieval. More important than the nature of the signal is that the foregoing explanation of signal-generated $\mathrm{N}$-length effects on resistance to extinction hinges on the assumption that rats can store a series of reinforcement events as a chunk and subsequently retrieve a memory of the entire chunk when presented with a signal that is extrinsic to the chunk. Recent experiments on chunking in serial learning tasks support this assumption (Capaldi, Miller, Alptekin, \& Barry, 1990; Haggbloom, Birmingham, \& Scranton, 1992).

\section{REFERENCES}

CAPAldi, E. J. (1966). Partial reinforcement: A hypothesis of sequential effects. Psychological Review, 73, 459-477.

Capaldi, E. J., Miller, D. J., Alptekin, S., \& Barry, K. (1990). Organized responding in instrumental learning: Chunks and superchunks. Learning \& Motivation, 21, 415-433.

HAGGBLOOM, S. J. (1988). The signal-generated partial reinforcement extinction effect. Journal of Experimental Psychology: Animal Behavior Processes, 14, 83-93.

haggbloom, S. J., Birmingham, K. M., Scranton, D. L. (1992). Hierachical organization of series information by rats: Series chunks and list chunks. Learning \& Motivation, 23, 183-199.

Haggbloom, S. J., Lovelace, L., Brewer, V. R., Levins, S. M., \& OWENS, J. D. (1990). Replacement of event-generated memories of nonreinforcement with signal-generated memories of reinforcemen during partial reinforcement training: Effect on resistance to extinction. Animal Learning \& Behavior, 18, 315-322.

(Manuscript received October 16, 1992.) 